\title{
Impactos da indústria 4.0 e sua autonomia operacional em processos de automação, através da internet das coisas
}

João Vitor Zanata de Souza joao.zanata@gmail.com

Faculdade de Tecnologia de Bebedouro (FATEC), Bebedouro, São Paulo, Brasil.

Luiz Rodrigo Bonette luiz.bonette@fatec.sp.gov.br

Faculdade de Tecnologia de Bebedouro (FATEC), Bebedouro, São Paulo, Brasil.

\author{
RESUMO
}

A indústria 4.0 no Brasil ainda é pouco difundida e explorada, isso faz com que a capacidade de produção das fabricas não são $100 \%$ exploradas e muito dinheiro é gasto sem previsão e sem informações suficientes, a intenção desse trabalho é demonstrar de maneira prática a diferença que um sistema de indústria 4.0 pode fazer em uma fábrica de laticínios, demonstrando os benefícios que a automação e que o Controle Estatístico do Processo (CEP) de qualidade podem trazer, a metodologia utilizada foi pesquisa-ação, foram 6 meses do ano de 2016 desde a primeira etapa de levantamento de informações até a finalização do projeto de implantação da Internet das Coisas (Internet of Things) no processo de automação das balanças de pesagem de uma indústria. As contribuições foram associadas a diminuição de perdas.

PALAVRAS-CHAVE: Indústria 4.0. IoT. Automação. Controle. Qualidade. 


\section{INTRODUÇÃO}

A indústria 4.0 é o futuro paradigma da produção onde muitas industrias ainda permanecem utilizando maquinário antigo e de operação manual, neste caso, a indústria 4.0 e a automação permite extrair informações e simplificando operações (KOLBERG; ZULKE, 2015). Schmitt (2015) esclarece que a indústria 4.0 destaca, por exemplo, o uso de dispositivos do tipo "smart" no chão de fábrica, isso possibilita a coleta de dados e informações nos quais dificilmente iria conseguir sem um investimento. No Brasil, o uso desses dispositivos é pequeno e, poucas pessoas e empresas possuem know how para utilização eficiente e lucrativa.

O potencial de Internet of Things (IOT) na produtividade brasileira e na melhoria dos serviços críticos do país poderia chegar a cerca de USD 200 bilhões por ano, em 2025, considerando a utilização de loT em todos os ambientes de aplicação, incluindo os ganhos econômicos diretos Produto Interno Bruto (PIB) e indiretos (consumer surplus) (RELATÓRIO FASE 1 - 3C DO BNDES, 2017).

O objetivo deste estudo visa analisar os impactos da indústria 4.0 no processo de pesagem de envase de manteiga através da inserção da loT, neste caso, seu desempenho e resultados operacionais em uma indústria na região de São Sebastião do Paraíso. O problema de pesquisa discute que as empresas cada vez mais sentem a necessidade de atualização passando por limitações adaptativas entre equipamentos automatizados e o processo incipiente a maturação da indústria 4.0 dentro, onde as empresas necessitam ter esta experimentação deste processo evolutivo industrial. Os processos de automação de equipamentos com base em inclusão de softwares com base nos fundamentos da Internet of Things (IOT) melhor e otimiza a indústria 4.0 em um segmento de tecnologia no Brasil?

Nota-se melhoria entre o processo de automação com a inclusão do software com base nos conceitos da loT e otimização do equipamento como possível hipótese da pesquisa.

Os processos de automação se tornam obsoletos a medida que os acessos a softwares são baixados de maneira gratuita aplicados aos equipamentos de produção, neste caso a contribuição é revelada que empresa brasileiras de tecnologia podem otimizar seus processos de produção através da junção e experimentação de inclusão de softwares com base na loT a melhoria de processos de automação em equipamentos com o auxílio da análise do desempenho. Este processo incipiente entre automação e inclusão de softwares com base nos processos de loT pode dar a tendência de melhoria dos processos através de elementos formadores do conceito da Industria 4.0.

O estudo de caso único dá suporte ao sequenciamento metodológico, sendo que a pesquisa é aplicada, com objetivo experimental entre o processo de automação e após isto implantação de softwares da loT.

O artigo é apresentado em sua estrutura pela revisão bibliográfica da Indústria 4.0 e a loT, processo de automação e Controle Estatístico do Processo (CEP), junto da composição do método de pesquisa-ação e o processo metodológico pela seção da metodologia, os principais fatores de impactos pelo desempenho e resultado entre automação e loT e o texto de Considerações 
Finais expondo os principais achados e contribuições futuras para a transição entre automação e Industria 4.0 utilizando IoT em processos de automação.

\section{REFERENCIAL TEÓRICO}

\section{INDÚSTRIA 4.0 E A INTERNET DAS COISAS}

IoT, sigla que vem do termo americano "Internet of Things" no português "Internet das Coisas", pode ser considerado o estado atual e futuro da tecnologia, mostrando que cada vez mais um dispositivo simples possa estar conectado à internet e consequentemente ao mundo. Esses dispositivos podem ser de diversas áreas, podendo trazer benefícios inimagináveis a nossa sociedade, contribuindo para que a informação seja compartilhada da maneira mais simples e rápida possível (FACHINI et al., 2017).

A Indústria 4.0 é uma nova forma de conectar produção, ferramentas e processos na internet através de sistemas físico-virtual, com capacidade de operar, coletar dados, interagir entre si e se auto corrigir de maneira autônoma se necessário (JUNIOR; SALTORATO, 2018).

Ao longo dos anos, o desenvolvimento dos mercados contribuiu para a formação de clientes mais exigentes, e isso se tornou um desafio para as indústrias no que se diz respeito a personalização dos produtos, alinhado a isso o baixo custo de produção e a eficiência para se manter competitiva no mercado (RODRIGUES et al., 2016). Um programa chamado Industrie 4.0 foi criado pelo governo alemão, ele junta investimentos governamentais e da própria indústria, para que, através disso todas suas áreas de seu processo produtivo sejam conectadas através de redes inteligentes, levando assim a produção industrial a realizar uma nova revolução industrial, no qual processos governam a si mesmo (RODRIGUES et al., 2016).

Em 2011, o ano no qual foi citado o termo Indústria 4.0, esse tema recebeu bastante atenção de governantes, empresários, universidades e pesquisadores, sendo um tema novo e ainda com pouca exploração, as pesquisas sobre Industria 4.0 e Internet das Coisas se revelaram muito abrangentes, praticamente um novo campo, com diversas possibilidades e caminhos, que veio sendo abordada de diversas áreas, como engenharia, administração e principalmente computação (JUNIOR E SALTORATO, 2018).

As três revoluções industriais foram acontecendo ao longo de 200 anos, e com elas mudanças nos processos produtivos ocorreram, essas mudanças foram estudadas após o acontecimento das mesmas, na quarta revolução industrial, a chama de indústria 4.0 pela primeira vez está sendo estudada e investigada antes de ocorrer e enquanto está acontecendo (PEREIRA, 2018).

Diferente de todas as revoluções passadas, a quarta revolução industrial não muda apenas a indústria, ela muda também a sociedade, política, setor público e a economia. Ela engloba tudo isso junto com inovações tecnológicas, tais como automação, controle, tecnologia da informação, coleta de dados, tudo isso através de loT (Internet of Things), Sistemas Cyber- Físicos e Internet dos Serviços (BRITO, 2017). 
A forma com que as revoluções industriais transformaram a vida das pessoas foi mudando ao longo dos anos, formas de energia alternativas, mais variedade de produtos, mais qualidade e quantidade de produtos, evoluções no processo de distribuição, sistema de transportes novos, tudo isso fez com que houvesse uma grande migração do campo para cidade (FILHO, 2016). Tudo isso agora estará interligado com a já ocorrente quarta revolução industrial, no qual vai mudar a forma com que as pessoas se relacionam, revolucionando o acesso a informação, gerando crescimento econômico, maior poder de compra, empregos mais qualificados e elevações do padrão de vida (FILHO, 2016).

Indústria 4.0 não é simplesmente um novo conceito, mas um conjunto de tecnologias e estudos que quando colocadas em sinergia proporcionam benefícios em diversas áreas da indústria, fazendo com que os equipamentos isolados se comuniquem entre si e forneça informações vitais para tomada de decisão (CARVALHO; FILHO, 2018).

\section{PROCESSO DE AUTOMAÇÃO}

Sistemas automáticos foram criados para facilitar a vida da população, mesmo que isso ocorra muitas das vezes em ambiente fabril, não estando tão visível para grande parte das pessoas que não tem acesso direto a uma fábrica (VILELA; VIDAL, 2003).

A automação ao longo do tempo vem melhorando e aprimorando o desempenho dos processos, de tal forma que é possível eliminar ou minimizar perdas, refugos, tempos improdutivos e processos, e colaborando para a padronização e qualidade dos produtos (SERVELIN et al., 2018). Vilela e Vidal (2003) explicam, por exemplo, que um processo fabril automatizado o benefício conseguir produzir com menor custo, maior quantidade, maior qualidade, menor tempo, conseguindo assim aumentar a produtividade sem comprometer prazos, preço e qualidade.

Segundo Vilela e Vidal (2003) explicam que, porém, a automação também pode ser utilizada em prol do meio ambiente, controlando as emissões de gases produzidos nas fabricas, líquidos que sobram do processo, utilização de materiais mais limpos, tudo isso de maneira simples e rápida.

Grandes mudanças aconteceram nesses anos referentes aos esquemas de produção, todos com o objetivo de aumentar a produção, disponibilidade, inovação, qualidade, diminuir custos e tempo. A automação junto a inteligência artificial e aos dispositivos loT tornam a indústria competitiva se destacando perante as outras (JUNIOR et al., 2003). Por outro lado, Vilela e Vidal (2003) descrevem que a automação está presente em diversas áreas ao redor com um único objetivo de facilitar processos.

Os sistemas de automação permitem controle e traz agilidade aos processos a serem feitos, essas tecnologias usam de Controladores Lógicos Programáveis (CLPs), permitindo assim que a pessoa que montou ele configure e desenvolva seu funcionamento através de programação (SILVEIRA et al., 2015).

Automação é a substituição do homem por máquinas, que possuem capacidade de mais produção veloz, gastando menos e sendo mais precisa, neste caso, novos problemas surgem fazendo uma constante evolução dos processos 
baseado no controle mecanizado até a mais atual das tecnologias, como microeletrônica (RONCOLI et al., 2015).

Diversas a essas empresas que buscam aumentar a produtividade, se destacando perante seus concorrentes e conseguindo entregar no mercado um produto de melhor qualidade, com menor custo e tempo, a automação industrial disponibiliza isso, com equipamento correto, especifico e devidamente configurado para finalidade de monitorar o processo de produção, e intervir quando algo sair do padrão, diminuindo o esforço humano necessário para tal tarefa e gerando uma rentabilidade satisfatória (SANTOS et al., 2017).

As ferramentas da qualidade vêm sendo aplicadas com mais frequência em diversas áreas de empresas que buscam melhorar seus produtos ou até mesmo uma certificação, a tecnologia de automação auxilia e facilita a empresa no controle de qualidade rigoroso e logo sendo indispensável (SOUZA et al., 2011).

A automação pode nem sempre ser utilizada apenas em indústrias, pois diversos edifícios da atualidade utilizam isso como uma forma de melhorar a interação entre pessoas e computadores, com o objetivo de fazer a gestão e controles de recursos em um prédio, para que o mesmo se tornasse o mais confortável e otimizada possível, para qual a finalidade seja melhorar a produtividade e facilitar as atividades dos mesmos, isso cada vez é uma realidade, visto que já existem residências que utilizam desses mesmos dispositivos, com o objetivo de facilitar a utilização de equipamentos domésticos, temperatura do ambiente, entre outros (OLIVEIRA et al.; 2016).

\section{CONTROLE ESTATÍSTICO DO PROCESSO DE QUALIDADE}

Souza et al. (2007) explicam que as empresas que fornecem seus produtos para diversos clientes ao redor do mundo enfrentam uma competitividade muito grande, por isso que investir em formas de se destacar no mercado acaba se tornando um diferencial, e um dos maiores diferenciais que se pode ter é a qualidade. A aplicação de controle estatístico de processo (CEP) pode trazer uma grande vantagem à empresa, se utilizada em produtos vistos como estratégicos, é possível identificar caminhos para melhorar a qualidade a ser percebida pelos clientes.

Lima et al. (2006) consideram o CEP como uma das mais poderosas formas que foram desenvolvidas com o objetivo de auxiliar o controle da qualidade, utilizando gráficos de controles ou cartas podemos detectar desvios de parâmetros, reduzindo assim a quantidade de produtos fora de especificações e consequentemente custos a produção.

A gestão da qualidade como uma função de gerência, uma disciplina acadêmica e uma área de pesquisa e desenvolvimento vem passando, ao longo do século passado, por aprimoramentos contínuos e gradativos. Após a II Grande Guerra, governos, especialistas e organizações tem empenhado para criar formas de aplicar ao planejamento, medição, análise, monitoramento, além de controle e melhoria da qualidade (REBELATO et al, 2008).

O controle estatístico de processo é uma ferramenta com uma grande utilidade, com ele se consegue incorporar o conceito de boas práticas de fabricação, também de fornecer informações muito importantes para validar os 
processos, pois através de investigações, pode-se analisar todos os pontos críticos e identificar aonde existe não conformidade nos processos de fabricação, possibilitando a correção dos mesmos (LIMA et al., 2006).

Rebelato et al. (2008) descrevem que a ausência de continuidade interativa em aplicações dos métodos de qualidade nas empresas é uma realidade, parte disso pode-se explicar que, para cara método e ferramenta foi criada em um período diferente, por empresas e organizações diferentes que no momento de sua criação, havia um problema pontual e seu objetivo era saná-la. Por isso que os métodos não possuem interfaces já previstas para aplicação com outros métodos, o que é relevante, porém a falta de integração dificulta a ação gerencial.

A velocidade das informações e que as novas tecnologias foram estabelecendo um ambiente competitivo e globalizado, no qual prazo, preço, confiabilidade e flexibilidade devem ser atendidos, o Controle Estatístico de Processos (CEP) auxilia nisso, pois, é uma ferramenta da qualidade que nos permite monitorar características de interesse, tais como citadas acima. Além de que nos permite assegurar a manutenção dentro de limites preestabelecidos e também indicando quando adotar ações de melhora e correções, os benefícios que essa ferramenta pode trazer para empresa são grandes, como o menor custo de produção, atendimento ao cliente e também maior competitividade no cenário em que ela se encontra (MIYATA et al. 2010).

Franken et al. (2013) explicaram que podem utilizar da análise estatística que, por sua vez resulta em informações, dados estatísticos que geram informações, essas informações podem ser de diversos equipamentos, processos, que se tornam muito importante pois, através da verificação desses dados podemos identificar possíveis falhas, erros e não conformidade. Além disso, ele serve também para garantir que os processos produtivos estejam e permaneçam com a qualidade desejada. A maioria de grandes empresas, indústrias, internacionais e nacionais investe massivamente em CEP para garantir a qualidade de seus produtos e para aumentar os níveis de produtividade e se manter competitiva no mercado em que atua.

Para conseguir atingir um nível de qualidade nos produtos, de tal forma que não cause a impossibilidade de utilização do mesmo pelo consumidor, é necessário evitar custos de não qualidade nos produtos, normalmente isso é relacionado a falhas na produção, podendo ser gerada por não utilização das ferramentas da qualidade ou por ineficiência. Conseguir a melhoria da qualidade junto ao processo de produção é e vem se tornando um desafio e ao mesmo tempo um diferencial para as empresas que o possuem. Conseguir sustentar um padrão de qualidade e que atenda as expectativas e requisitos dos clientes é um diferencial que podem citar com a utilização do CEP, além de que ao identificar falhas e ineficiência nos processos, podemos trabalhar para diminuir os custos de produção e manter bons índices de produtividade (ADAMY et al, 2015).

\section{METODOLOGIA}

O ciclo da investigação-ação se divide em dois "setores" e quatro fases, sendo os setores de investigação e ação. $E$ as fases sendo designadas em planejar, agir, descrever e analisar. A maioria dos ciclos de melhoria segue da 
mesma forma, é identificado o problema, planejado a solução após isso é realizado a implementação e a avaliação, para saber se foi feito de forma correta e o problema foi solucionado (TRIPP, 2005).

Consegue-se diferenciar a pesquisa-ação de outros tipos de investigaçãoação definindo a mesma pelas técnicas que são empregadas nas pesquisas, a fim de produzir a descrição dos efeitos das mudanças. Quando é utilizado o termo "investigação-ação" como um processo, a mesma inclui a pesquisa-ação, que acaba se tornando sem sentido, pois está sendo aplicada de maneira vaga (TRIPP, 2005).

Neste caso a pesquisa-ação foi sequenciada nas etapas abaixo:

- Investigar e diagnosticar uma situação prática ou um problema que precisa ser melhorado ou resolvido;

- Formular estratégias de ação;

- Desenvolver essas estratégias e avaliar a sua eficiência;

- Ampliar a compreensão dessa nova situação

Prosseguir os mesmos passos para uma nova situação prática.

\section{ANÁLISE DE RESULTADOS E DISCUSSÃO}

\section{ANÁLISE DOS RESULTADOS}

Nota-se acima, a Industria 4.0, internet of things (IoT) e o Controle Estatístico do Processo de qualidade podem ajudar e muito uma empresa a ter lucro e ser competitivo no mercado, a seguir temos algumas imagens do centro de monitoramento de uma fábrica de laticínios, ela investiu em tecnologias que a ajudassem a controlar melhor a fabricação de seu produto, que no caso é manteiga. A coleta de dados foi aplicada a pesagem dos potes de manteiga, cada máquina envasa 2 potes de manteiga por vez que passam por uma balança, o pote de 200 gramas precisa ter por lei $2 \%$ a mais em seu conteúdo, a máquina que realiza a pesagem mostrava apenas o peso atual do pote em questão, portanto você não conseguiria saber com exatidão qual o peso médio dos potes para regular as máquinas por exemplo

Figura 1 - Envase da manteiga Fonte: Elaborado pelos autores (2019)



Fonte: Autoria Própria (2019) 
Quando os dados começaram a ser medidos e comparados, via-se que estavam perdendo muita manteiga, pois a máquina estava envasando 213 gramas (Figura 2) ao invés de 204 que seria o (Figura 3).

Figura 2 - Média do desempenho com 213 gramas Fonte: Elaborado pelos autores (2019)

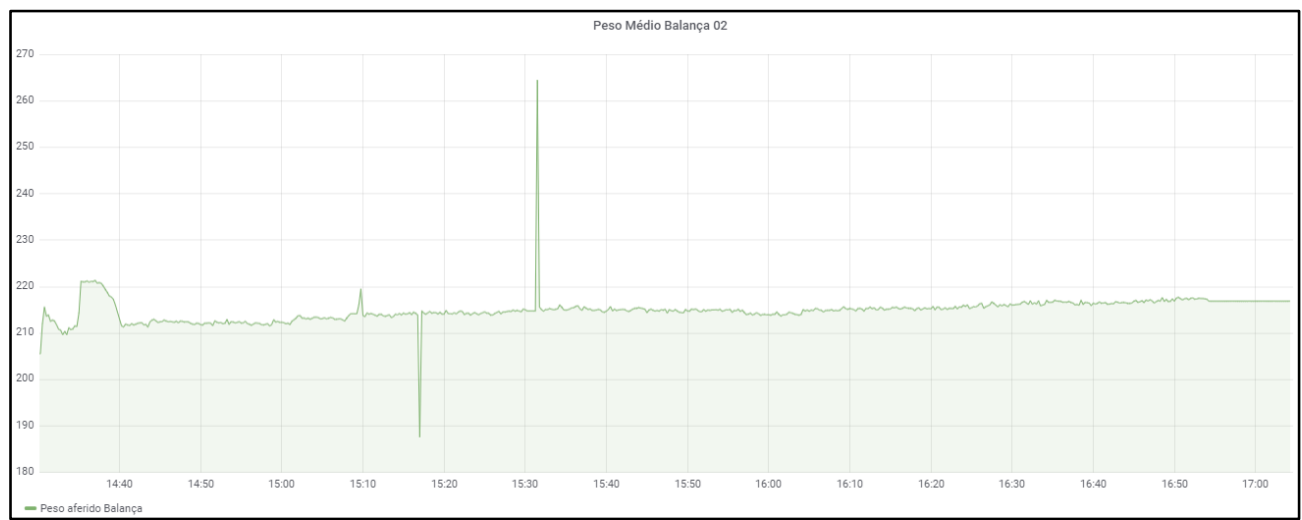

Figura 3 - Média do desempenho com 204 gramas Fonte: Elaborado pelos autores (2019)

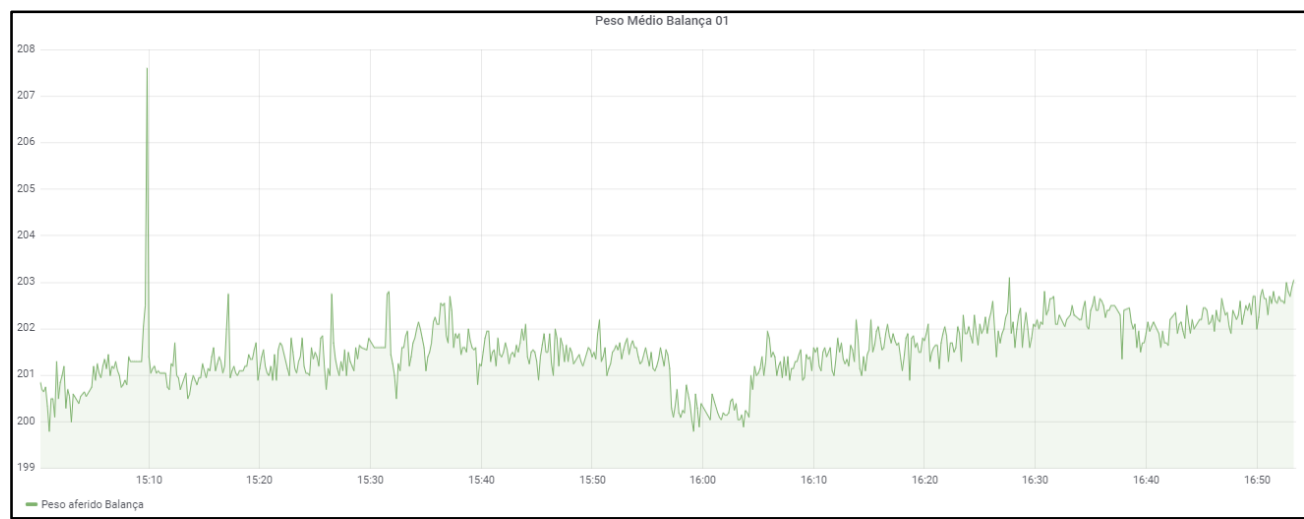

Através dessa medição, é possível ver e regular melhor as máquinas para que o desperdício seja evitado, aumentando assim o lucro, porém não é apenas para isso que serve o loT, nessa mesma fábrica é monitorado diversos outros equipamentos e status tais como na figura 4.

Figura 4 - Dashboard

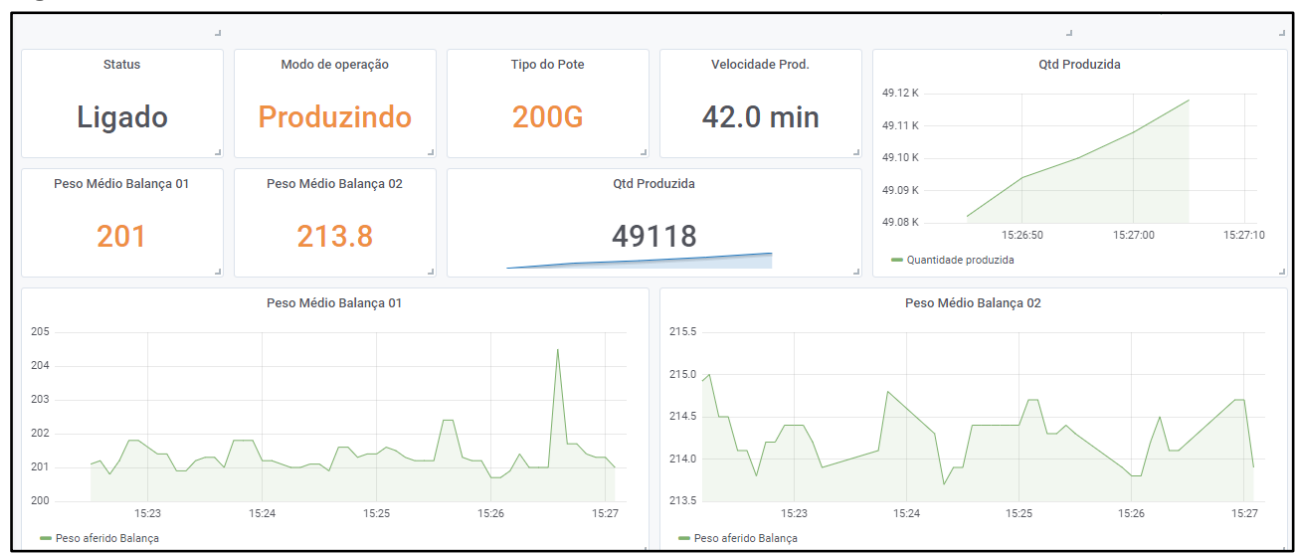

Fonte: Autoria Própria (2019) 
Podem-se analisar nesse processo do dashboard as seguintes informações:

- Status da máquina;

- Modo de operação (Produzindo, Limpeza ou Esperando);

- Tipo do Pote (200g ou 500g);

- Velocidade de produção;

- Quantidade produzida (Gráfico);

- Peso médio das balanças (Quantitativo);

- Quantidade produzida (Quantitativo);

- Peso médio das balanças (Gráfico).

Isso pode ser colocado em um display no chão de fábrica para verificar status das máquinas, conferir se pesagem está correta, se a quantidade do lote está correta, ou então numa sala de reuniões em tempo real para acompanhar e comparar gráficos de produções passadas, saber quantidades produzidas por hora, dia, mês, ano. Pode ser acessado via smartphone pelo CEO da empresa, onde ele estiver e saber os dados da produção.

\section{DISCUSSÃO}

É percebido que o processo metodológico por etapas do método de pesquisa ação associado análise do processo de implantação da tecnologia loT na pesagem da balança resultou nas seguintes observações:

- A busca pela empresa do cliente em investigar e diagnosticar essa situação prática ou o problema que precisava ser melhorado ou resolvido sobre a troca do processo antigo sobre o novo, nesta etapa o cliente percebeu que existia um processo de perda nos volumes dos equipamentos visualmente na relação produto e preenchimento da embalagem (pote), porém não sabia o quanto, pois não existia a mensuração da perda e do seu critério a verificar na prática pela CEP proposta, e isso dificultou a atratividade pelo processo e a aquisição de futuros investidores.

- Foram formuladas estratégias de ação sequenciadas em:

Primeira etapa: Levantamento das informações relevantes a serem coletadas e analisadas por diversos protótipos que poderiam ser aplicados ao processo com tempo de duração de 1 mês.

Segunda etapa: Desenvolvimentos dos protótipos dos equipamentos utilizados para extração das informações da planta com a duração de 1 mês no processo de análise.

Terceira etapa: Implementação dos equipamentos com a duração de 2 meses

Quarta etapa: Coleta de dados e posteriormente a análise dos mesmos em parceira e aplicabilidade com o cliente com a duração de 2 meses.

Ao todo foram 6 meses entre levantamento, protótipos, implementação e testes. O sistema é constantemente monitorado pela empresa de tecnologia de implantação de tecnologias de Indústria 4.0 sendo o fornecedor, neste caso o sistema sofre atualizações constantes, recentes e mensais. 
Após a última etapa se apropriando dos dados, os mesmos geraram os insights necessários e assim decisões de economia foram geradas, essas ações foram:

- Melhor acompanhamento mão de obra no sistema de envase.

- Aprimorar a tecnologia de envase para que houvesse maior precisão no processo. Essa última decisão foi tomada apenas após a análise dos dados, pois após isso eles obtiveram os dados necessários para analisar se seria um bom investimento ou não, visto que seria um investimento de grande porte.

Desta maneira o processo e implantação da loT na automação das balanças de pesagem ampliou a compreensão dessa nova situação e prosseguiu os mesmos passos para uma nova situação prática, pois houve eficiência no processo, e o mesmo procedimento foi repedido na segunda máquina de envase também.

\section{CONCLUSÃO}

A loT e a Industria 4.0 percorrem o mesmo caminho em busca da otimização dos processos, juntas e sendo bem aplicadas podem render diversos benefícios para empresa, não só na qualidade, mas também no lucro, regulando da forma correta as máquinas para que não excedem o mínimo permitido de $2 \%$, a fábrica conseguiu produzir mais, com a mesma quantidade de matéria prima.

Essa é apenas um exemplo do que pode ser feito caso seja implementado corretamente com as ferramentas certas e com a equipe certa, existem outras fábricas operando sistemas parecidos com funcionalidades diversas, inclusive armazéns com monitoramento de temperatura, umidade, gasto de energia entre outros. Contribui para pesquisa futuras o estudo contínuo do método da pesquisa ação para implantação de tecnologias da Indústria 4.0 em outros processos do segmento de laticínios ou da produção e logística agroindustrial no Brasil. 


\title{
Impacts of Industry 4.0 and its operational autonomy in automation processes, through the Internet of things
}

\begin{abstract}
Industry 4.0 in Brazil is still not widespread and exploited, this makes the production capacity of factories are not $100 \%$ exploited and a lot of money is spent without forecast and without enough information, the intention of this work is to practically demonstrate the difference. that a 4.0 industry system can do in a dairy factory, demonstrating the benefits that automation and quality Statistical Process Control (CEP) can bring, the methodology used was action research, were 6 months of 2016 from the first stage of information gathering to the completion of the Internet of Things deployment project in the process of automating the weighing scales of an industry. Contributions were associated with decreased losses.
\end{abstract}

KEYWORDS: Industry 4.0. IOT. Automation. Control. Quality. 


\section{REFERÊNCIAS}

ADAMY, A. P. A; GOMES, T. E. O.; STORCK, O.; ZANINI, R. R.; ROSA, L. C. O uso do controle estatístico de processo como forma de garantia de qualidade para o cliente: aplicação em uma indústria metalmecânica. Revista ESPACIOS. 2015.

BRITO, A. A. F. B. A Quarta Revolução Industrial e as Perspectivas para o Brasil. Revista Científica Multidisciplinar Núcleo do Conhecimento. Edição 07. Ano 02, Vol. 02. P.91-96. 2017. crossref

CARVALHO, E. S. S.; FILHO, N. D. F. Proposta de um sistema de aprendizagem móvel com foco nas características e aplicações práticas da indústria 4.0. RISTI, N. 27. Instituto Federal de Educação, Ciência e Tecnologia de São Paulo, Sertãozinho, Brasil. 2018. crossref

FACHINI, P. M.; MESQUITA, P. N.; OLIVEIRA, P. R.; FRANÇA, G. P. Internet das Coisas: Uma Breve revisão Bibliográfica. Conex. Ci. e Tecnol. Faculdade de Informática e Administração Paulista. Fortaleza/CE, v.11, n. 6, p. 85-90. 2017. crossref

FRANKEN, D. A.; HAMMES, J.; POLACINSKI, E.; LORENZETT, D. B.; GODOY, L. P.; GODOY, T. P. O controle estatístico de processos em uma empresa de agronegócios. Rev. ADM. UFSM, Santa Maria, RS. 2013. crossref

FILHO, J. R. H. A era da internet industrial e a indústria 4.0. Revista Eletrônica Produção em Foco. v.6, n.3 - Joinville, SC. 2016.

JÚNIOR, A. P. A.; CHAGAS, C. V.; FERNANDES, R. G. Uma rápida análise sobre automação industrial. Redes para Automação Industrial - 2003.1. DCA-CTUFRN. Natal - RN. 2003.

JUNIOR, G. T.; SALTORATO, P. Impactos da Indústria 4.0 na organização do trabalho: Uma revisão sistemática da literatura. Revista Produção Online. Florianópolis, SC, v. 18, n. 2, p. 743-769. 2018. crossref

KOLBERG, D.; ZÜHLKE, D. Lean auto-mation enabled by industry 4.0 technologies. Kaiserslautern: IFAC. 2015. crossref

LIMA, A. A. N; LIMA, J. R.; SILVA, J. L.; ALENCAR, J. R. B.; SOARES-SOBRINHO, J. L.; LIMA, L.G; ROLIM-NETO, P. J. Aplicação do controle estatístico de processo na indústria farmacêutica. Revista de Ciências Farmacêuticas Básica e Aplicada. UFRJ, Rio de Janeiro, RJ. 2006. 
MIYATA, H. H.; BARRETO, A.; GOMES, A. C. S.; MORAIS, M. F.; ROCHA, R.

Peterson. Controle estatístico de processo na produção de circuitos eletrônicos.

XXX Encontro Nacional de Engenharia de Produção. Anais...São Carlos, SP, 2010.

OLIVEIRA, A. S.; MOTTA, R. A. S. M.; CUNHA, G. G. Percepções sobre o inoctopus: Uma ferramenta para facilitar a interação entre pessoas e tecnologias na gestão e controle de construções futuristas e de pesquisa. XXVI SIMPETP. Anais...Bauru, SP, 2016.

PEREIRA, A. Indústria 4.0: Conceitos e Perspectivas para o Brasil. Revista da Universidade Vale do Rio Verde. ISSN: 1517-0276 / EISSN: 2236-5362 Vol. 16, n. 1, 2018. crossref

REBELATO, M. G.; FERNANDES, J. M. R.; RODRIGUES, A. M. Proposta de integração entre métodos para planejamento e controle da qualidade. Revista Gestão Industrial. UTFPR, Ponta Grossa, PR. 2008. crossref

RELATÓRIO FASE 1 - 3C BNDES (2017). Disponível em: https://www.bndes.gov.br/wps/wcm/connect/site/6c597bfe-b92d-4084-ab075498e1ae2445/produto-3-analise-de-oferta-e-demanda-relatorio-parcial-analisede-demanda.pdf?MOD=AJPERES\&CVID=ISZJkHO Acesso em: 18 abr. 2019.

RODRIGUES, F. L.; JESUS, A. R.; SCHUTZER, K. Industrie 4.0 - Uma Revisão da Literatura. Revista de Ciência \& Tecnologia. Universidade Metodista de Piracicaba, SP, v. 19, n. 38, p. 33-45. 2016. crossref

RONCOLI, M. A; LANZOTTI, P. H. A Automação como ferramenta de melhoria em um processo produtivo. Faculdade de Tecnologia de Taquaritinga (FATEC) - SP Brasil, 2015.

SANTOS, A. C.; OLIVEIRA, B. A.; JUNKES, V. H.; PEDROSO, M. A. A. M.; GOMES, I. F. Automação industrial em pequenas, médias e grandes empresas: Um estudo teórico. XXXVII Encontro nacional de Engenharia de Produção. Anais...Joinville, SC, 2018.

SCHMITT, M.; ORFGEN, M.; ZULKE, Detlef Dynamic Reconfiguration of Intelligent Field Devices by using Modular Software Applications. Kaiserlautern: IFAC, 2015. crossref

SERVELIN, T.; MARAFON, C.; ANSCHAU, C. T.; SCHNEIDER, A.; PAULA, R. 
Benefícios do investimento em automação no processo de empacotamento de farinha de trigo. Anais... - Engenharia de Produção - ISSN - 2594 - 4657 V.2. №1, 2018.

SILVEIRA, T.; SILVA, A. L. E.; MORAES, J. A. R.; BRUM, T. M. M. Proposta de automação para o processo de fabricação de borrachas escolares. XI Congresso Nacional de Excelência em Gestão. Anais...Rio de Janeiro - RJ, 13 e 14/08 2015.

SOUZA, J.; WILBORN, F.; KALSHNE, N. F. Uso da Automação para controle da qualidade na indústria. Revista arbitrada de gestion tecnológica. Espacios. v.34, ed.10. 2013.

SOUZA, G. P.; FILHO, M. D.; SMOHYL, W. W. Aplicação dos conceitos de Controle Estatístico de Processo (CEP) em uma indústria de fundição do Norte Catarinense. Revista Produção On Line. Florianópolis - SC - Brasil. 2007. crossref

TRIPP, D.; Pesquisa-ação: uma introdução metodológica. Educ. Pesquisa. v.31 n.3. São Paulo Sept/Dec. 2005. crossref

VILELA, P. S. C.; VIDAL, F. J. T. Automação Industrial. Redes para Automação Industrial - DCA- 2401 UFRN. Maio de 2003.

DE SOUZA, J.V.Z. et al. Impactos da industria 4.0 e sua autonomia operacional em processos de automação, através da internet das coisas. R. Gest. Industr., Ponta Grossa, v. 15, n. 4, p. 42-55, Out./Dez. 2019. Disponível em: http://periodicos.utfpr.edu.br/revistagi. Acesso em: 2019. Correspondência: João Vitor Zanata de Souza

Faculdade de Tecnologia de Bebedouro (FATEC), Bebedouro, São Paulo, Brasil.

Direito autoral: Este artigo está licenciado sob os termos da Licença Creative Commons-Atribuição 4.0 Internacional.

\section{(c) (1)}

